\title{
Progression of late gadolinium enhancement in a young patient with $\mathrm{HCM}$
}

\author{
Sebastian Estrada, Pelbreton Balfour and Christopher M Kramer* \\ University of Virginia Health System, Charlottesville, VA, USA
}

\begin{abstract}
Hypertrophic cardiomyopathy (HCM) is the most common genetic heart disease, affecting 1 in 500 individuals. It is characterized by unexplained myocardial hypertrophy with the underlying pathology of myofibrillar disarray and interstitial fibrosis. Complications of this disease include sudden cardiac death due to ventricular tachyarrhythmias and heart failure, both of which may be related, at least in part to the underlying fibrosis in the myocardium.

Cardiac magnetic resonance imaging (CMR) is the only imaging technique available to identify myocardial fibrosis in vivo with the technique of late gadolinium enhancement. Both the presence and extent of LGE have been shown to be associated with adverse cardiac outcome in HCM. However, few studies have examined progression of LGE in HCM. Little is known about the initial development of LGE. In this case report, we present a case of development of LGE in a young adult with HCM.
\end{abstract}

\section{Case report}

A 22-year-old male presented in 2012 to the UVA Hypertrophic Cardiomyopathy (HCM) Center of Excellence after being previously diagnosed with suspected HCM after a routine sports physical. At the time of evaluation, he was otherwise asymptomatic without a history of shortness of breath, chest pain, orthopnea, palpitations, or syncope. Of note, family history revealed a maternal uncle who suffered sudden cardiac death in his 50's. Physical examination revealed a mid to late systolic murmur with a grade of $1 / 6$, with intensity increasing with Valsalva [1-5].

EKG showed sinus rhythm and left ventricular hypertrophy by voltage criteria with secondary repolarization abnormalities. Transthoracic echocardiogram (TTE) demonstrated moderate asymmetric septal hypertrophy with normal LV cavity size and ejection fraction of $75 \%$ with, mild mitral regurgitation and systolic anterior motion (SAM), but no significant outflow gradient. Exercise stress testing was unremarkable. Holter monitor showed sinus rhythm with no premature ventricular contractions and rare premature atrial contractions. No high grade tachycardia or bradycardia arrhythmias were recorded. Genetic testing revealed no known clinically significant variants in the common genes linked to HCM. CMR showed asymmetric septal hypertrophy with a septal:posterior wall ratio of 1.6:1, SAM, and mild mitral regurgitation. No evidence of late gadolinium enhancement (LGE) was identified (Figure1A-B). In 2014, 2 years after his initial visit and CMR, he was enrolled in the HCMR study [6] and underwent a research CMR where evidence of LGE in the $\mathrm{RV}$ insertion sites was noted (Figure1C-D).

\section{Discussion}

HCM is generally caused by mutations in genes that encode cardiac sarcomere proteins that are most commonly inherited in an autosomal dominant fashion. Patients diagnosed at a younger age tend to have a higher risk of sudden cardiac death whereas patients diagnosed later in life tend to have comparable mortality to the general public [7].

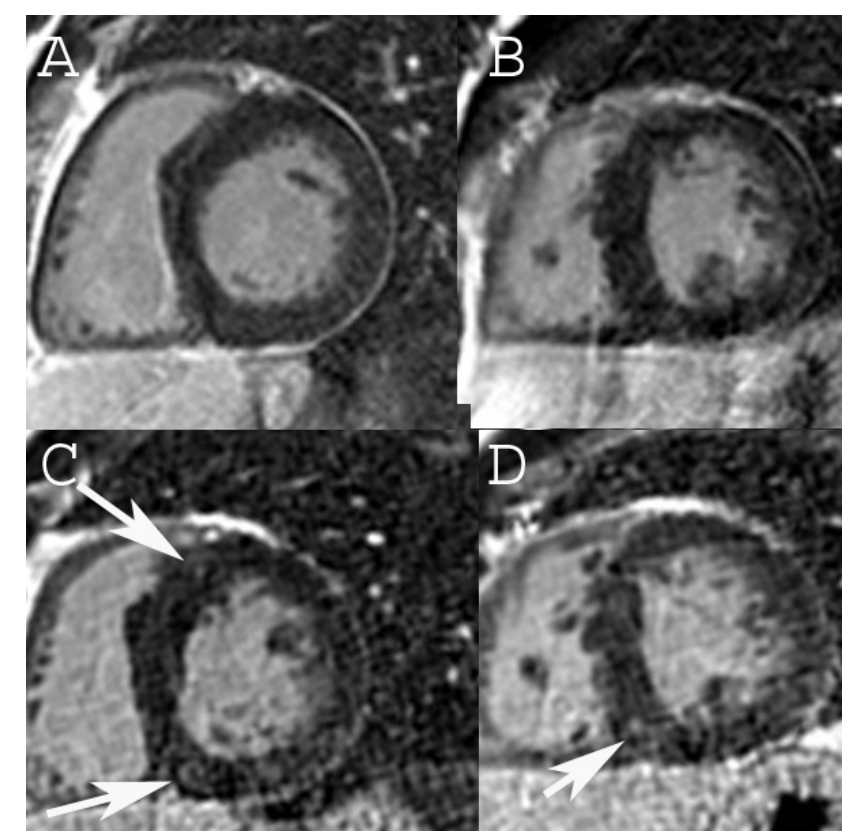

Figure 1. CMR from 2013 in the base (A) and mid- LV (B) showing no LGE. CMR from 2014 shows LGE in the anteroseptal (C) and inferoseptal RV insertion site (C, D).

Although echocardiography is the typical method used for screening and diagnosis, CMR has emerged as the gold standard for imaging morphology and fibrosis with both LGE (replacement fibrosis)

${ }^{*}$ Correspondence to: Christopher M Kramer, University of Virginia Health System, Departments of Medicine and Radiology, Lee Street, Box 800170, Charlottesville, VA 22908, USA, Tel: (434) 243-0736; Fax: (434) 982-1998; E-mail: ckramer@virginia.edu

Received: June 15, 2018; Accepted: June 22, 2018; Published: June 27, 2018 
and T1 mapping (interstitial fibrosis) [8]. The presence of LGE has been associated with cardiac death. There is growing evidence that the extent of LGE corresponds to the risk of SCD [3]. Effective risk stratification is vital for identifying the patients that would benefit most from an implantable cardioverter defibrillator to prevent fatal ventricular arrhythmias.

This particular case highlights the early development and progression of LGE in a young patient. Two previous studies have documented progression of LGE in patients $[4,5]$ but the patients were much older on average. The study by Todiere et al demonstrated that of $55 \mathrm{HCM}$ patients with a mean age of $43 \pm 18$ at baseline, 45 had some LGE [4]. Of the 10 that had none, 8 developed LGE over the ensuing 2 years. In the study by Choi et al, the mean age was even higher ( $59 \pm$ 13) and the prevalence of LGE was $91.5 \%$ at the first CMR. While there are no current guidelines for serial CMR studies in HCM, the reported case shows that progression from no to some fibrosis is possible over a relatively short time frame such as 2 years and the transition from no fibrosis to some fibrosis may occur in the early 20 's. Tracking progression of fibrosis in patients with HCM could shed further light on the natural history of this disease and the potential relationship to adverse events.

\section{References}

1. Maron BJ, Ommen SR, Semsarian C, Spirito P, Olivotto I, et al. (2014) Hypertrophic cardiomyopathy: present and future, with translation into contemporary cardiovascular medicine. J Am Coll Cardiol 64: 83-99. [Crossref]

2. Weng Z, Yao J, Chan RH, He J, Yang X, et al. (2016) Prognostic Value of LGE-CMR in HCM: A Meta-Analysis. JACC Cardiovasc Imaging 9: 1392-1402. [Crossref]

3. Chan RH1, Maron BJ1, Olivotto I1, Pencina MJ1, Assenza GE, et al. (2014) Prognostic Value of Quantitative Contrast-Enhanced Cardiovascular Magnetic Resonance for the Evaluation of Sudden Death Risk in Patients With Hypertrophic Cardiomyopathy. Circulation 130: 484-495. [Crossref]

4. Todiere G1, Aquaro GD, Piaggi P, Formisano F, Barison A, et al. (2012) Progression of Myocardial Fibrosis Assessed With Cardiac Magnetic Resonance in Hypertrophic Cardiomyopathy. J Am Coll Cardiol 60: 922-929. [Crossref]

5. Choi HM, Kim KH, Lee JM, Yoon YE, Lee SP, et al. (2015) Myocardial fibrosis progression on cardiac magnetic resonance in hypertrophic cardiomyopathy. Heart 101: 870-876. [Crossref]

6. Kramer CM, Appelbaum E, Desai MY, Desvigne-Nickens P, DiMarco JP, et al. (2015) Hypertrophic Cardiomyopathy Registry: The rationale and design of an international, observational study of hypertrophic cardiomyopathy. Am Heart $J$ 170: 223-230. [Crossref]

7. Maron BJ, Rowin EJ, Casey SA, Garberich RF, Maron MS (2016) What Do Patients With Hypertrophic Cardiomyopathy Die from? Am J Cardiol 117: 434-435. [Crossref]

8. Maron MS, Maron BJ (2015) Clinical Impact of Contemporary Cardiovascular Magnetic Resonance Imaging in Hypertrophic Cardiomyopathy. Circulation 132: 292 298. [Crossref]

Copyright: (C2018 Estrada S. This is an open-access article distributed under the terms of the Creative Commons Attribution License, which permits unrestricted use, distribution, and reproduction in any medium, provided the original author and source are credited. 